\title{
Parametric Resonance of Optically Trapped Aerosols
}

\author{
R. Di Leonardo, ${ }^{1, *}$ G. Ruocco, ${ }^{1}$ J. Leach, ${ }^{2}$ M. J. Padgett, ${ }^{2}$ A. J. Wright, ${ }^{3}$ J. M. Girkin, ${ }^{3}$ \\ D. R. Burnham, ${ }^{4,5}$ and D. McGloin ${ }^{4,5}$ \\ ${ }^{1}$ INFM-CRS SOFT c/o Universitá di Roma "La Sapienza," I-00185, Roma, Italy \\ ${ }^{2}$ SUPA, Department of Physics and Astronomy, University of Glasgow, Glasgow, Scotland, United Kingdom \\ ${ }^{3}$ Institute of Photonics, SUPA, University of Strathclyde, Glasgow, Scotland, United Kingdom \\ ${ }^{4}$ SUPA, School of Physics and Astronomy, University of St. Andrews, St. Andrews, Scotland, United Kingdom \\ ${ }^{5}$ Electronic Engineering and Physics Division, University of Dundee, Dundee, Scotland, United Kingdom
}

(Received 23 February 2007; published 5 July 2007; corrected 6 July 2007)

\begin{abstract}
The Brownian dynamics of an optically trapped water droplet are investigated across the transition from over- to underdamped oscillations. The spectrum of position fluctuations evolves from a Lorentzian shape typical of overdamped systems (beads in liquid solvents) to a damped harmonic oscillator spectrum showing a resonance peak. In this later underdamped regime, we excite parametric resonance by periodically modulating the trapping power at twice the resonant frequency. The power spectra of position fluctuations are in excellent agreement with the obtained analytical solutions of a parametrically modulated Langevin equation.
\end{abstract}

DOI: 10.1103/PhysRevLett.99.010601

Parametric resonance provides an efficient and straightforward way to pump energy into an underdamped harmonic oscillator [1]. If the resonance frequency of an oscillator is dependent upon a number of parameters, modulating any of these at twice the natural oscillation frequency parametrically excites the resonance. Such behavior leads to surprising phenomena in the macroscopic world (pumping a swing, stability of vessels, surface waves in vibrated fluids) [2,3]. On the microscopic scale, where stochastic forces become important, one refers to Brownian parametric oscillators [4]. The parametric driving of Brownian systems has been shown to be at the origin of some peculiar behaviors such as the squeezing of thermal noise in Paul traps [5]. Parametrically excited oscillations have also been reported in a single-crystal silicon microelectromechanical system [6]. What makes parametric resonance useful is that it is easier to modulate a system parameter rather than to apply an oscillating driving force. Moreover, for finite but low damping rates, one never reaches a stationary state with the damping forces dissipating the input power, and, consequently, the amplitudes of oscillations diverge. Optically trapped microparticles constitute a beautiful example of a Brownian damped harmonic oscillator, and they are becoming an increasingly common tool for the investigation of different fields of basic and applied science [7]. Pumping mechanical energy into optically trapped particles could open the way to many applications. In optical tweezers, even though it is easy to periodically modulate the laser power, parametric excitation is usually ineffective because of the heavy damping action of the surrounding fluid.

It has been reported that modulating the laser power at the parametric resonant frequency in an overdamped system increased the amplitude of fluctuations [8,9]. However, these results have been difficult to reproduce and are in
PACS numbers: 05.40.-a, 46.40.Ff, 82.70.Rr, 87.80.Cc

contrast to the predictions of the Langevin equation [1012].

In this Letter, we demonstrate how parametric resonance can be excited in optically trapped water droplets suspended in air. We measure power spectra of position fluctuations and find an excellent agreement with the theoretical expectations based on Langevin dynamics. Besides providing a resolution of a still-controversial issue, our results are the first study of the dynamics of trapped particles in a gas-damped tweezers system, a configuration that is finding wider applications in the study of aerosol droplets and associated atmospheric chemistry [13].

The dynamics of an optically trapped droplet is described by the Langevin equation [14]:

$$
\ddot{x}(t)+\Omega_{0}^{2} x(t)+\Gamma_{0} \dot{x}(t)=\xi(t),
$$

where $\Omega_{0}^{2}=k / m$ is the angular frequency of the oscillator depending on trap stiffness $k$ and particle mass $m$. $\Gamma_{0}=$ $6 \pi \eta a / C_{c} m$ is the viscous damping due to the medium viscosity $\eta$ and depending on particle radius $a$ and mass $m$. To correct Stokes' law for finite Knudsen number effects, we introduced the empirical slip correction factor $C_{c}$, with a $5.5 \%-1.6 \%$ reduction in drag for $3-10 \mu \mathrm{m}$ diameter droplets, respectively [15].

The stochastic force $\xi$, due to thermal agitation of solvent molecules, is generally assumed to have a white noise power spectrum:

$$
S_{\xi}(\omega)=\frac{\Gamma_{0} K_{B} T}{\pi m} .
$$

By Fourier transforming (1) and using (2), we can easily obtain the power spectrum of position fluctuations [16]:

$$
S_{x}(\omega)=\frac{K_{B} T}{k} \frac{1}{\pi} \frac{\Omega_{0}^{2} \Gamma_{0}}{\left(\omega^{2}-\Omega_{0}^{2}\right)^{2}+\Gamma_{0}^{2} \omega^{2}} .
$$


The ratio $\Gamma_{0} / \Omega_{0}$ depends only slightly on particle size and, in solvents with waterlike viscosities, is always greater than 1 (the system is overdamped) up to power levels of some tens of watts. For typical trapping powers of order $10 \mathrm{~mW}$ in water, $\Gamma_{0} / \Omega_{0}$ is typically $>10$. As a result, only those frequencies smaller than $\Gamma_{0}$, and hence much smaller than $\Omega_{0}$, have a significant amplitude in the power spectrum. Under these conditions, we can therefore neglect $\omega^{2}$ with respect to $\Omega_{0}^{2}$ in the first term of the denominator in (3) and obtain the usual Lorentzian power spectrum characterized by $\omega^{-2}$ tails [16]. Such an overdamped condition precludes the possibility of exciting significant oscillations either directly or parametrically. To probe oscillations in the liquid-damped regime, we would need to be able to increase typical trap power by 4 orders of magnitude. A more feasible route is to reduce viscosity by 2 orders of magnitude, which can be readily obtained by trapping particles in air whose viscosity is approximately $1 / 55$ of water $\left(\eta=1.8 \times 10^{-5} \mathrm{Pas}\right)$ [15].

Our optical tweezers are based around an inverted microscope with a high numerical aperture oil immersion microscope objective $(1.3 \mathrm{NA}, 100 \times)$. The continuous wave laser is a Nd:YAG, frequency doubled to give $0-2 \mathrm{~W}$ of $532 \mathrm{~nm}$ light. To couple the beam into the air medium, a single cover slip is rested over the objective on a thin oil layer. In a method similar to Refs. [17,18], a water aerosol is produced using a nebulizer $(3.4-6.0 \mu \mathrm{m})$ and injected into a sample chamber $30 \mathrm{~mm}$ in diameter and $10 \mathrm{~mm}$ deep, sealed by the cover slip. This isolates the droplets from convective air currents and create a near-saturated atmosphere within which the droplet size is stable. To obtain a saturated atmosphere, we decrease the vapor pressure of the droplets by adding salt to the water. In such conditions, the droplet quickly reaches an equilibrium size between condensation and evaporation and has a size stability within $2 \%$ over the trapping time [19]. After a few seconds, a droplet in the range of 4-7 $\mu \mathrm{m}$ is trapped at the beam focus; see Fig. 1. For our laser powers, this gives a trap resonance frequency in the vicinity of $2 \mathrm{kHz}$, and we can maintain the trap for $40 \mathrm{~min}$. For particles trapped in fluid, a laser power of $10 \mathrm{~mW}$ is typical; however, to maximize the stiffness of our traps (and hence $\Omega_{0}$ ), we use powers of $100 \mathrm{smW}$. Despite this, we calculate a temperature increase of less than $1 \mathrm{~K}$ due to laser heating [17]. Though this does not significantly enhance evaporation, temperature gradients across the droplet, due to nonuniform heating, could initiate thermal Marangoni effects. However, being concerned with the center of mass dynamics occurring in the kilohertz region, none of these relatively slow phenomena disturbs the high frequency dynamics. A quadrant photodetector, placed in the back focal plane of the condenser lens, receives the light transmitted through the droplet. By measuring the imbalance of the light collected by the quadrants, the lateral displacement of the droplet is deduced with a bandwidth of several kilohertz and a precision of better than $5 \mathrm{~nm}$ [20]. The

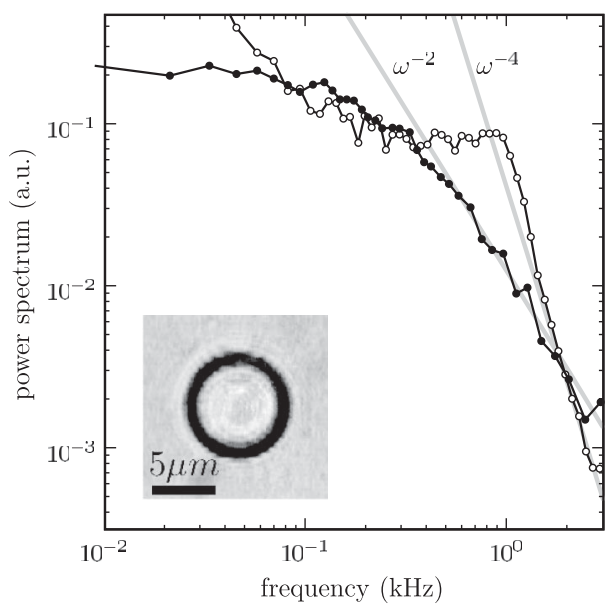

FIG. 1. The measured power spectra of trapped aerosol particle at two different powers. At lower power (black circles), it is overdamped, and the mean squared amplitude of the high frequency motion decays as $\omega^{-2}$. At higher powers (white circles), the aerosol is underdamped, and the mean squared amplitude decays as $\omega^{-4}$. Gray lines show the calculated slopes -2 and -4 . The inset shows an optical image of a trapped aerosol particle.

stability of our system allows a series of power spectra to be obtained from the same droplet while scanning one parameter. The three reported experimental protocols (Figs. 1-3) will refer to three independently trapped droplets.

The power spectra of the measured displacement, for two different trap powers, are shown in Fig. 1. It is clear how the particle dynamics changes from an overdamped Lorentzian spectrum with a high frequency roll-off proportional to $\omega^{-2}$ to an underdamped regime with a faster roll-off $\omega^{-4}$ and the appearance of a resonance peak near $1 \mathrm{kHz}$ (more clearly seen in Fig. 2, which is plotted on a linear scale). The emergence of such a peak arises from the

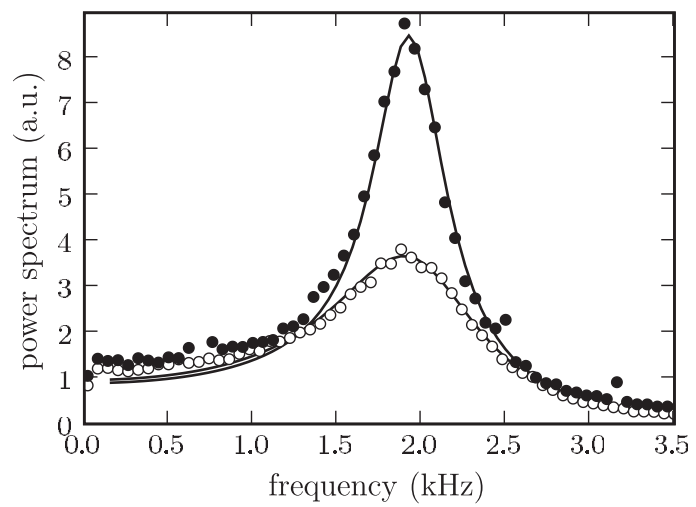

FIG. 2. The measure power spectrum of a trapped water droplet for no modulation of the laser power (white circles) and modulation at $3.9 \mathrm{kHz}\left(\Omega_{1} \simeq 2 \Omega_{0}\right)$ (black circles). The peak is higher and narrower on the resonant condition, thus indicating parametric excitation. The solid line below the black circles is the predicted spectrum from (11). 


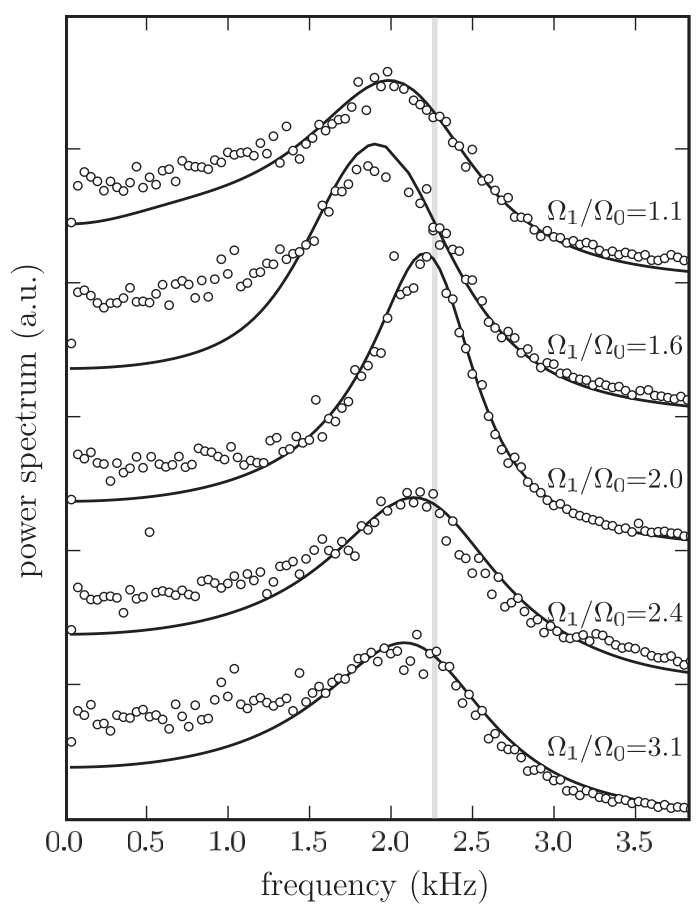

FIG. 3. Evolution of position power spectra on varying the modulation frequency $\Omega_{1}$. Parametric excitation of oscillations is evident at the parametric resonance condition $\Omega_{1} / \Omega_{0}=2$. The solid lines are the theoretical predictions from (11). The vertical gray line indicates $\Omega_{0} / 2 \pi=2.3 \mathrm{kHz}$.

fact that the inertial terms in (1) are no longer negligible. As a consequence, an average trajectory starting away from the equilibrium position crosses the equilibrium position with a finite velocity. In this situation, the parametric resonance is excited by modulating the strength of the trapping potential. Ideally, the potential is made shallower when the particle traverses the equilibrium position and steeper again when the particle is far from the equilibrium position. This is maximally efficient when we modulate the potential at twice the natural oscillation frequency $\Omega_{0}$. To consider this model in quantitative terms, we can rewrite (1) in the presence of a parametrically modulated external potential:

$$
\begin{gathered}
\ddot{x}(t)+\Omega_{0}^{2}[1+g f(t)] x(t)+\Gamma_{0} \dot{x}(t)=\xi(t), \\
f(t+\mathcal{T})=f(t), \quad-1<f(t)<1,
\end{gathered}
$$

where $0<g<1$ measures the strength of modulation. By Fourier transforming (4), we obtain

$$
\begin{aligned}
& \left(-\omega^{2}+\Omega_{0}^{2}+i \omega \Gamma_{0}\right) \hat{x}(\omega) \\
& +\Omega_{0}^{2} g \sum_{k=-\infty}^{\infty} a_{k} \hat{x}\left(\omega+k \Omega_{1}\right)=\hat{\xi}(\omega),
\end{aligned}
$$

where $a_{k}$ is the coefficient of the $k 2 \pi / \mathcal{T}=k \Omega_{1}$ frequency component of the Fourier series expansion of $f(t)$. It is clear from Eq. (6) how parametric modulation introduces a coupling between all of those frequencies differing by an integer number of $\Omega_{1}$. We now introduce the vectors $X_{n}(\omega)=\hat{x}\left(\omega+n \Omega_{1}\right) \quad$ and $\quad R_{n}(\omega)=$ $\hat{\xi}\left(\omega+n \Omega_{1}\right)$ and write the recursive relations:

$$
\begin{aligned}
{\left[-\left(\omega+n \Omega_{1}\right)^{2}+\Omega_{0}^{2}+\right.} & \left.i\left(\omega+n \Omega_{1}\right) \Gamma_{0}\right] X_{n}(\omega) \\
& +\Omega_{0}^{2} g \sum_{k=-\infty}^{\infty} a_{k} X_{n+k}(\omega)=R_{n} .
\end{aligned}
$$

To obtain the power spectrum $S_{x}(\omega)$, for each frequency $\omega$ we compute $X_{0}(\omega)$. This is coupled to all other components in the array $X_{n}$. However, the strength of the coupling will decay for large $|n|$, so that we can limit ourselves to a finite number of components and write the matrix equation for the array $\mathbf{X}(\omega)=\left[X_{-N}(\omega), \ldots, X_{N}(\omega)\right]$ :

$$
\mathbf{G}^{-1}(\omega) \mathbf{X}(\omega)=\mathbf{R}(\omega),
$$

with

$$
\begin{aligned}
G_{n k}^{-1}(\omega)= & {\left[-\left(\omega+n \Omega_{1}\right)^{2}+\Omega_{0}^{2}+i\left(\omega+n \Omega_{1}\right) \Gamma_{0}\right] \delta_{n k} } \\
& +\Omega_{0}^{2} g a_{k-n} .
\end{aligned}
$$

By matrix inversion, we obtain the power spectrum as

$$
\begin{aligned}
\left\langle X_{0}^{*}(\omega) X_{0}\left(\omega^{\prime}\right)\right\rangle & =\sum_{k, n=-N}^{N} G_{0 k}^{*}(\omega) G_{0 l}\left(\omega^{\prime}\right)\left\langle R_{k}^{*}(\omega) R_{n}\left(\omega^{\prime}\right)\right\rangle \\
& =\frac{\Gamma_{0} K_{B} T}{\pi m} \sum_{n=-N}^{N}\left|G_{0 n}(\omega)\right|^{2} \delta\left(\omega-\omega^{\prime}\right),
\end{aligned}
$$

and from the definition of power spectrum:

$$
S_{x}(\omega)=\frac{\Gamma_{0} K_{B} T}{\pi m} \sum_{k=-N}^{N}\left|G_{0 k}(\omega)\right|^{2} .
$$

If $\Omega_{0}$ and $\Gamma_{0}$ are known, we can use (11) to predict the power spectrum of a droplet in a modulated trap. The white circles in Fig. 2 show the measured power spectrum for a water droplet trapped with constant laser power. The presence of the peak suggests that we are in an underdamped regime. By fitting these data to Eq. (3), we can deduce the resonant frequency $\Omega_{0} / 2 \pi=2.0 \mathrm{kHz}$ and the damping term $\Gamma_{0}=6.8 \mathrm{kHz}$ for our experimental conditions. The fitted value of $\Gamma_{0}$ corresponds to the Stokes drag on an aerosol droplet of radius $3.4 \mu \mathrm{m}$. We then apply the square-wave modulation of the trapping power adjusted to give the same average power as before, $\Omega_{1} \simeq 2 \Omega_{0}$ and $g=0.4$. The black circles in Fig. 2 show the corresponding power spectrum of the motion. As expected, the resonance is excited, matching closely the expected behavior obtained by applying the measured parameters $\Omega_{0}, \Gamma_{0}, \Omega_{1}$, $g$ to Eq. (11), supporting our interpretation of a parametric excitation of the resonance. Using these parameters with (11), we can make further predictions about the dynamics that can be verified by our observations. One comparison is the predicted and observed form of the power spectra as a function of the modulation frequency, both above and 


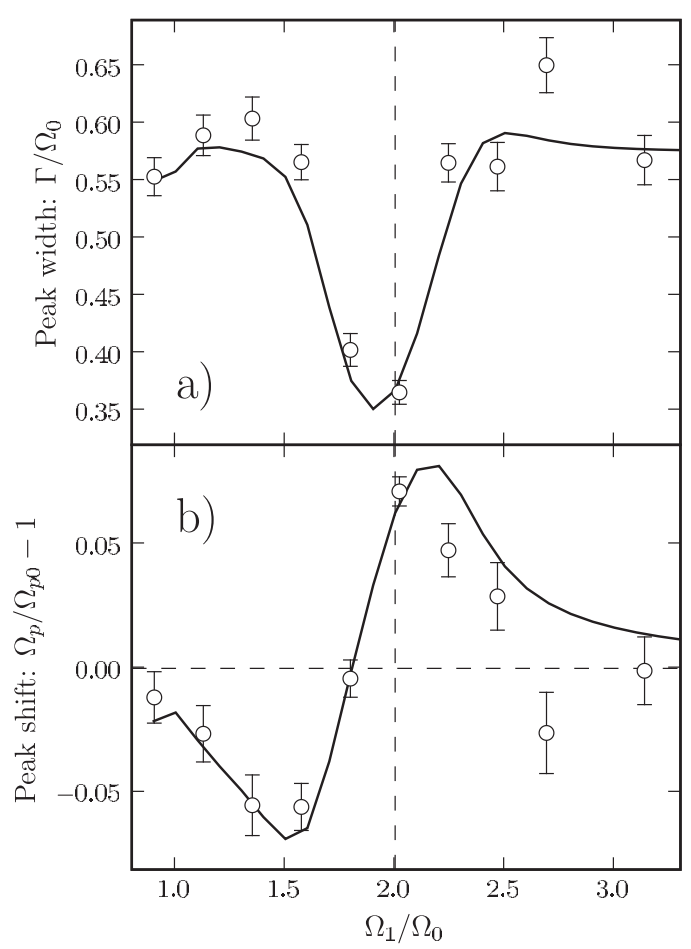

FIG. 4. (a) Peak full width at half maximum as a function of modulation frequency. The solid lines are the theoretical predictions from (11). (b) Peak position shift as a function of modulation frequency. $\Omega_{p 0}$ is the peak position in the absence of modulation.

below the parametric resonance condition; see Fig. 3 . Again, there is an excellent agreement between the observed and predicted particle motions. In particular, the parametric excitation of oscillations manifests as a narrowing of the peak (or a reduced apparent damping $\Gamma$, defined as the full width at half maximum), occurring when modulating at twice $\Omega_{0}$. Close to parametric resonance, a shift in peak position $\Omega_{p}$ is also apparent. Both of these signatures confirm our interpretation of the system as being a Brownian parametric oscillator; see Fig. 4.

We recognize that our observations relate to the study of the lateral motion of the trapped droplets. In keeping with other work, we note from examination of the video images that the axial movement of the trapped droplet occurs on much longer time scales, corresponding to frequencies in the subkilohertz region. This slow axial dynamics is responsible for the low frequency component of the spectra in Fig. 3. This reflects the comparatively weak axial trapping, possibly arising from aberrations associated with nonoptimized objectives. It may be possible to use dough- nut or Laguerre-Gaussian beams having zero on-axis intensity and improved axial trapping [21].

We have reported the first observation of a parametrically excited resonance within a Brownian oscillator. The demonstration of this effect within optical tweezers was made possible by relying on the low viscosity of air to lightly damp the motion of a trapped aerosol droplet. The detailed observed dynamics matches closely the power spectra predicted from a parametrically modulated Langevin equation.

We thank S. Ciuchi for many helpful discussions. D. M. thanks the Royal Society for support.

*roberto.dileonardo@phys.uniroma1.it

[1] C. Van den Broeck and I. Bena, in Stochastic Processes in Physics, Chemistry and Biology (Springer-Verlag, Berlin, 2000).

[2] L. Ruby, Am. J. Phys. 64, 39 (1996).

[3] J. Bechhoefer and B. Johnson, Am. J. Phys. 64, 1482 (1996).

[4] C. Zerbe, P. Jung, and P. Hänggi, Phys. Rev. E 49, 3626 (1994).

[5] A. F. Izmailov, S. Arnold, S. Holler, and A. S. Myerson, Phys. Rev. E 52, 1325 (1995).

[6] L. Turner et al. Nature (London) 396, 149 (1998).

[7] D. G. Grier, Nature (London) 424, 810 (2003).

[8] J. Joykutty, V. Mathur, V. Venkataraman, and V. Natarajan, Phys. Rev. Lett. 95, 193902 (2005).

[9] V. Venkataraman, V. Natarajan, and N. Kumar, Phys. Rev. Lett. 98, 189803 (2007).

[10] L. Pedersen and H. Flyvbjerg, Phys. Rev. Lett. 98, 189801 (2007).

[11] Y. Deng, N. R. Forde, and J. Bechhoefer, Phys. Rev. Lett. 98, 189802 (2007).

[12] Y. Deng, J. Bechhoefer, and N. R. Forde, J. Opt. A Pure Appl. Opt. (to be published).

[13] J. Buajarern, L. Mitchem, A. D. Ward, N. Nahler, D. McGloin, and J.P. Reid J. Chem. Phys. 125, 114506 (2006).

[14] S. Chandrasekhar, Rev. Mod. Phys. 15, 1 (1943).

[15] J.H. Seinfeld and S. N. Pandis, Atmospheric Chemistry and Physics: Air Pollution to Climate Change (Wiley, New York, 1997).

[16] M. C. Wang and G. E. Uhlenbeck, Rev. Mod. Phys. 17, 323 (1945).

[17] R. J. Hopkins, L. Mitchem, A. D. Ward, and J. P. Reid, Phys. Chem. Chem. Phys. 6, 4924 (2004).

[18] D. R. Burnham and D. McGloin, Opt. Express 14, 4175 (2006).

[19] L. Mitchem et al., J. Phys. Chem. A 110, 8116 (2006).

[20] F. Gittes and C. Schmidt, Opt. Lett. 23, 7 (1998).

[21] A. T. O’Neil and M. J. Padgett, Opt. Commun. 193, 45 (2001). 